\title{
Bifidobacterium longum bv. infantis CCUG 52486 combined with gluco-oligosaccharide significantly reduces the duration of self-reported cold and flu-like symptoms among healthy older adults after seasonal influenza vaccination
}

\author{
C. E. Childs ${ }^{1}$, A. Przemska ${ }^{1}$, C. Maidens ${ }^{1}$, H. Dong ${ }^{1}$, I. Bin Dayel ${ }^{1}$, A. A. Fekete ${ }^{1}$, I. R. Rowland ${ }^{1}$, \\ K. M. Tuohy ${ }^{2}$, S. C. Todd ${ }^{1}$, M. A. Gosney ${ }^{1}$ and P. Yaqoob ${ }^{1}$ \\ ${ }^{1}$ Department of Food and Nutritional Sciences, University of Reading RG6 6AP and ${ }^{2}$ Nutrition and Nutrigenomics Group, \\ Research and Innovation Centre-Fondazione Edmund Mach, San Michele all'Adige, Trento, Italy
}

The potential health benefits of both pre- and probiotics have expanded in recent years from maintaining bowel regularity and a balance of gut microflora to improving micronutrient status and immune function. There is particular interest in the positive influences of pre- and probiotics in older people, who are subject to alteration in gut microbiota composition and also to immunosenescence (deterioration and dysregulation of the immune system).

The PRIMAGE study is a randomised, double-blind, placebo-controlled, parallel study investigating the influence of Bifidobacterium longum $b v$. Infantis CCUG $52486\left(5 \times 10^{8} \mathrm{CFU} / \mathrm{d}\right)$ combined with gluco-oligosaccharide $(8 \mathrm{~g} / \mathrm{d})$ on the immune response to influenza vaccination among healthy young $(18-35 \mathrm{y}, n=58)$ and older $(60-85 \mathrm{y}, n=54)$ volunteers. Volunteers consumed either a placebo $(9 \mathrm{~g} / \mathrm{d}$ maltodextrin) or treatment for a total of 8 weeks, and a trivalent influenza vaccination (2010/2011 northern hemisphere) was administered after 4 wks of treatment. Blood and faecal samples were collected at baseline, week 4 and after vaccination. Older volunteers were asked to complete a self-reported illness form for six months post-vaccination.

43 healthy older volunteers were vaccinated in October/November 2010 in accordance with UK NHS vaccination schedules, and 41 returned a six-month self-reported illness form. Treatment did not significantly alter the incidence of cold or flu-like symptoms. Three participants within the treatment group reported a sudden onset of flu like illness, resulting in a significantly higher duration of this symptom among volunteers on treatment in the six months post-vaccination $(p=0.0047)$. Volunteers receiving treatment had significantly lower cumulative duration of fatigue, runny nose, headache and sore throat symptoms during the six months post-vaccination compared to placebo $(p<0.0001)$.

\section{Cumulative symptom duration (days)}
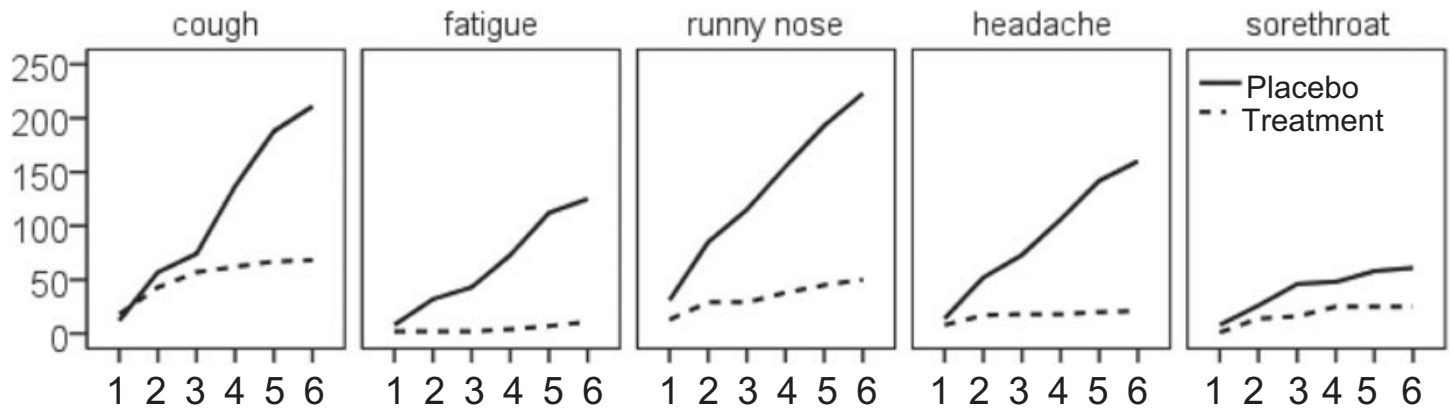

Month post vaccination

Data indicate that a discrete period of treatment with Bifidobacterium longum $b v$. Infantis CCUG 52486 combined with glucooligosaccharide can significantly mitigate the duration of winter cold and flu-like symptoms among healthy older adults. Further research is required to elucidate the immunological basis of these changes in volunteer-reported outcomes. 